\title{
POLYNOMIAL BOUNDS FOR SOLUTIONS TO BOUNDARY VALUE AND OBSTACLE PROBLEMS WITH APPLICATIONS TO FINANCIAL DERIVATIVE PRICING
}

\author{
LOUIS BHIM
}

(Received 1 September 2017; first published online 2 November 2017)

2010 Mathematics subject classification: primary 65C99; secondary 65K15, 90C22, $91 \mathrm{G} 80$.

Keywords and phrases: obstacle problem, optimal stopping, dual approach, free-boundary problem, polynomial bounds, American option price, regime switching.

Obstacle problems are of great theoretical and practical interest across a range of fields. Indeed, they are a classical motivating example in the study of variational inequalities and free-boundary problems which find applications in, for instance, fluid filtration in porous media, elastoplasticity, optimal control, financial mathematics and image processing. Intuitively, the classical obstacle problem seeks to describe the equilibrium state of an elastic membrane stretched over a solid obstacle and fixed at the boundary of the problem domain. In this sense, it can be thought of as an energy minimisation problem and, indeed, in its original study it was formulated as a constrained minimisation problem. Further formulations include the use of variational inequalities and linear complementarity conditions (see, for instance, [4, 8]).

In spite of these problems being well studied, they are rarely amenable to analytical solution and so numerical approaches are often necessary. Many numerical methods for approximating solutions to obstacle problems have been proposed over the years and they often assume one of the aforementioned formulations in order to develop their numerical scheme. In this thesis, we assume a different formulation of the problem and take a different and, in some sense, complementary approach to numerical solution of the obstacle problem. It is well known that the obstacle problem is a type of freeboundary problem and it has been shown that these problems can be formulated as an optimal stopping problem (see, for instance, [1]), the optimal stopping time being the first hitting time of a diffusion with a free boundary. Using this type of characterisation of the solution to an obstacle problem, we extend an optimisation approach for finding and tightening polynomial bounds for solutions to Dirichlet boundary value problems

Thesis submitted to the University of Sydney in January 2017; degree approved on 14 March 2017; supervisor Reiichiro Kawai.

(c) 2017 Australian Mathematical Publishing Association Inc. 0004-9727/2017 \$16.00 
(see, for instance, $[6,7]$ ) to the general framework of elliptic and parabolic obstacle problems and further to bound solutions to systems of boundary value problems and obstacle problems. The focus of this thesis is to derive the key results that justify the use of this approach, explore the properties of the bounds obtained and demonstrate the scope of the applications, particularly in the context of financial derivative pricing.

Chapter 1 presents the context of the study and Chapter 2 formulates elliptic and parabolic boundary value problems, presenting existing results for obtaining polynomial bounds for the solutions to these problems and discussing the scope of their applications, with reference to problems in financial derivative pricing.

Chapter 3 contains the main theoretical contributions of the thesis, extending the results presented in Chapter 2 to the more general framework of elliptic and parabolic obstacle problems. Here, the relevant underlying theory is developed, including desirable convergence results associated with the resulting bounds, and properties of these bounds are discussed. In particular, instead of obtaining approximations to the solution in the usual sense, we search for minimal supersolutions to act as tight upper bounds for the solution to the obstacle problem. That is, we identify a class of supersolutions to the obstacle problem and proceed to formulate and numerically solve an appropriate optimisation problem. The approach is then not only unique to existing methods from the point of view of the form of the bounds produced, but also at the level of implementation in terms of the type of numerical scheme utilised. The optimisation problem is a semidefinite programming problem, which is made numerically tractable using sum-of-squares relaxations and solved numerically using the semidefinite program solving software SDPT3 [11] and modelling language YALMIP [9]. The effectiveness of this method is demonstrated extensively through numerical examples.

It is well known that the problem of pricing American-style options can be formulated as an obstacle problem and, in general, has no explicit solution. Chapter 4 then demonstrates the applicability of the current approach to obtaining upper bounds on American option price functions. Interesting relationships are also derived between the proposed approach and existing methods for approximating American option prices (see, for instance, [5, 10]). Throughout this chapter, the effectiveness of the proposed method in obtaining tight upper bounds for American-style option prices is demonstrated in a variety of market models and with various payoff structures, such as the multivariate Black-Scholes and Heston stochastic volatility models and the American put and butterfly payoff structures. Extensions of the proposed methodology to perpetual American-style options and frameworks in which the underlying asset contains jumps are also discussed. The numerical examples of this chapter also highlight the features of the proposed approach that make it a practical alternative to existing methods. In particular, in a single implementation this approach obtains explicit bounds in the form of piecewise polynomial functions, which bound the price function from above over the whole problem domain in both time and state. As a consequence, these global bounds store a continuum of information in the form of a finite number of polynomial coefficients. The proposed approach achieves these 
bounds, free from statistical error, without recourse to sample path simulation, without truncating the naturally unbounded domain that arises in this problem and without discretising the time and state variables. This can be seen as a practical alternative to existing Monte Carlo approaches, which in one implementation may obtain a single statistical estimate to an upper or lower bound for the price at a single point in the problem domain. The results of Chapter 4 form the manuscript [2].

Finally, Chapter 5 extends this methodology for obtaining and tightening bounds for the solutions to boundary value and obstacle problems to bound solutions to systems of boundary value and obstacle problems. This is done by considering how one can extend the proposed approach to address problems in financial derivative pricing in regime-switching market models wherein the market dynamics change according to a continuous-time Markov process on a finite state space. These problems can be formulated as systems of coupled boundary value and obstacle problems and the theoretical and practical concerns that must be considered when extending the techniques of Chapters 2 and 3 to such problem settings are discussed. The results of Chapter 5 can be found in [3].

\section{References}

[1] A. Bensoussan and J.-L. Lions, Applications of Variational Inequalities in Stochastic Control (North-Holland, New York, 1982).

[2] L. Bhim and R. Kawai, 'Smooth upper bounds for the price of American style options', Int. J. Theor. Appl. Finance (2017), to appear.

[3] L. Bhim and R. Kawai, 'Polynomial upper and lower bounds for financial derivative price functions under regime-switching', J. Comput. Finance 22(2) (2018), to appear.

[4] A. Friedman, Variational Principles and Free Boundary Problems (Wiley-Interscience, New York, 1982).

[5] M. B. Haugh and L. Kogan, 'Pricing American options: a duality approach', Oper. Res. 52(2) (2004), 258-270.

[6] R. Kawai, 'Explicit hard bounding functions for boundary value problems for elliptic partial differential equations', Comput. Math. Appl. 70(12) (2015), 2822-2837.

[7] R. Kawai and K. Kashima, 'On weak approximation of stochastic differential equations through hard bounds by mathematical programming', SIAM J. Sci. Comput. 35(1) (2013), A1-A25.

[8] D. Kinderlehrer and G. Stampacchia, An Introduction to Variational Inequalities and Their Applications (Academic Press, London, 2000).

[9] J. Löfberg, 'Pre- and post-processing sum-of-squares programs in practice', IEEE Trans. Automat. Control 54(5) (2009), 1007-1011.

[10] L. C. G. Rogers, 'Monte Carlo valuation of American options', Math. Finance 12 (2002), 271-286.

[11] R. H. Tütüncü, K. C. Toh and M. J. Todd, 'Solving semidefinite-quadratic-linear programs using SDPT3', Math. Program. Ser. B 95 (2003), 189-217.

\section{LOUIS BHIM, School of Mathematics and Statistics,}

University of Sydney, NSW 2006, Australia

e-mail: louis.bhim@gmail.com 